\title{
Fabrication and evaluation of an all solid redox capacitor with an iodine based gel polymer electrolyte
}

\author{
C.M. Bandaranayake, W.A.D.S.S. Weerasinghe, K.S. Perera* and K.P. Vidanapathirana \\ Department of Electronics, Faculty of Applied Sciences, Wayamba University of Sri Lanka, Kuliyapitiya.
}

\begin{abstract}
Gel polymer electrolytes (GPEs) have demonstrated a greater potential to be used as electrolytes for various applications such as batteries, super capacitors, electrochromic devices and dye sensitised solar cells. They consist of a salt and a mixture of solvents trapped in a polymer matrix. In this study, optimisation and characterisation of a GPE consisting of polyvinylidene fluoride, ethylene carbonate, propylene carbonate and sodium iodide and its application in a redox capacitor with two polypyrrole electrodes was studied. GPE shows a conductivity of $9.69 \times 10^{-3} \mathrm{Scm}^{-1}$ at room temperature $\left(28{ }^{\circ} \mathrm{C}\right)$ with good mechanical stability. The corresponding composition is $1.13 \mathrm{PVdF}: 2.5 \mathrm{EC}: 2.5 \mathrm{PC}: 0.4 \mathrm{NaI}$ (by weight). The variation of conductivity with temperature follows Arhenius behaviour suggesting that the conductivity mechanism takes place via hopping of ions. Conductivity is purely ionic in nature. Properties of the redox capacitors were studied by the cyclic voltammetry (CV) technique, electrochemical impedance spectroscopy (EIS) technique and galvanostatic charge-discharge (GCD) test. The $\mathrm{CV}$ results showed the dependency of specific capacitance on the scan rate. The EIS results showed that capacitive behaviour becomes dominant only at low frequency range. The resulting specific capacitance was $3.19 \mathrm{Fg}^{-1}$. It was found that the redox capacitor exhibits an average discharge specific capacitance of $5.93 \mathrm{Fg}^{-1}$ while having a specific energy density of $0.03 \mathrm{Whkg}^{-1}$ and a specific power density of $0.42 \mathrm{Wkg}^{-1}$.
\end{abstract}

Keywords: Bode plot, cyclic voltammetry technique, gel polymer electrolyte, redox capacitor.

\section{INTRODUCTION}

There has been a rapid growth in research and development in the area of energy storage devices all over the globe. Supercapacitor, a new type of reversible electrochemical energy storage device, also known as an ultra capacitor has been identified as a very attractive device for energy storage. It has aroused an enormous research interest due to its fast energy delivery, short charging time, high power capability, long durability and environment-friendly features ( $\mathrm{Yu}$ et al., 2012). Supercapacitors possess higher capacitance than those achievable with conventional capacitors and can operate at substantially higher specific power than batteries (Tripathi et al., 2013). These have various consumer and industrial applications such as cellular communication devices, memory protection of computer electronics, hybrid automobiles and uninterruptible power supplies (Wang et al., 2013; Jayathilake et al., 2014a).

There are two types of supercapacitors which differ in the energy storage mechanism involved, namely, electric double-layer capacitor (EDLC) and redox capacitor (Singu et al., 2014). The EDLCs use carbon based electrodes. In redox capacitors, either metal oxide or conducting polymers such as polypyrrole, polyaniline, polythiophene etc., are used as the electrode material. Earlier, liquid electrolytes have been used commonly for redox capacitors but at present, attention has been diverted towards gel polymer electrolytes (GPEs) due to their attractive characteristics such as being free of internal shorting, no leakage and higher ionic conductivity (Jayathilaka et al., 2014b). GPEs which consist of a salt and solvent mixtures trapped in a polymer matrix possess high ionic conductivity and appreciable mechanical properties at ambient temperatures. For many of the

* Corresponding author (kumudu31966@gmail.com; (iD https://orcid.org/0000-0003-4491-662X) 
redox capacitors investigated so far, polymers such as polymethylmethacrylate (PMMA), polyacrylonitrile (PAN) and polyvinylidene-co-hexafluoropropylene (PVdF-HFP) have been used extensively to prepare GPEs (Pandey et al., 2010; Gupta \& Tripathi, 2013; Bandaranayake et al., 2016).

This paper describes a redox capacitor based on a GPE prepared using polyvinylidene fluoride $(\mathrm{PVdF})$, ethylene carbonate (EC), propylene carbonate (PC) and sodium iodide (NaI). PVdF is a less studied polymer for redox capacitors. There are two reasons for selecting $\mathrm{NaI}$ as the salt. Firstly, it is highly considered for dye sensitised solar cells (Khanmirzaei et al., 2015); secondly, it has been reported that smaller anions in the electrolyte give rise to higher capacities (Gupta \& Tripathi, 2013). For the characterisation of redox capacitors, cyclic voltammetry $(\mathrm{CV})$ technique, electrochemical impedance spectroscopy (EIS) and galvanostatic charge discharge test (GCD) were used.

\section{METHODOLOGY}

\section{Preparation of the gel polymer electrolyte}

Polyvinylidene fluoride (PVdF) (Aldrich, Mw-534 000), ethylene carbonate (EC) (Aldrich, $98 \%$ ), propylene carbonate (PC) (Aldrich, $99 \%$ ) and sodium iodide (NaI) (Aldrich, $99 \%$ ) were used to prepare the GPE film with the hot press method (Jayathilake et al., 2015). The EC and $\mathrm{PC}$ weight ratio was fixed as 1:1. First, PVdF and $\mathrm{NaI}$ were dissolved in the EC:PC mixture and magnetic stirring was done for $1 \mathrm{~h}$ until a homogenous mixture was formed. The mixture was heated at $120{ }^{\circ} \mathrm{C}$ for 45 mins. The hot mixture was pressed in between two well-cleaned glass plates. After separating the glass plates, it was possible to obtain a thin, free standing film. Several samples were prepared with different PVdF and $\mathrm{NaI}$ concentrations to optimise the composition to get the highest room temperature conductivity and mechanical stability.

\section{AC conductivity measurements}

A circular shape sample of diameter $14 \mathrm{~mm}$ was cut from the thin electrolyte film and it was sandwiched in between two well cleaned stainless steel (SS) electrodes in a spring loaded sample holder. The impedance measurements were taken in the frequency range of $0.01 \mathrm{~Hz}$ to $0.4 \mathrm{MHz}$ from $28^{\circ} \mathrm{C}$ to $55^{\circ} \mathrm{C}$ using Metrohm M101 impedance analyser. Thickness of the circular shape films was measured using a micrometer screw gauge. Conductivity of the GPE, $\sigma$, was calculated from the equation, $\sigma=\left(1 / R_{\mathrm{b}}\right) l / A$

where, $l$ is the thickness of the GPE film and $A$ is the area of the GPE film. The bulk electrolyte resistance, $R_{\mathrm{b}}$ was calculated using the resultant impedance plot and non linear least square fitting method.

\section{DC polarisation test}

DC polarisation test was done to find the ionic transference number $\left(t_{\mathrm{i}}\right)$ of the GPE sample. In this characterisation method, SS electrodes were used as blocking electrodes. A circular shape GPE sample was loaded in between two SS electrodes in a sample holder. Under a fixed $1 \mathrm{~V} \mathrm{DC}$ voltage, the current was monitored as a function of time. $t_{\mathrm{i}}$ of the GPE was calculated according to the relationship,

$t_{\mathrm{i}}=\left(I_{\mathrm{t}}-I_{\mathrm{s}}\right) / I_{\mathrm{t}}$

where $I_{\mathrm{t}}$ is the maximum current and $I_{\mathrm{s}}$ is the steady state current.

\section{Fabrication of redox capacitors}

Using a 3 electrode setup, the monomer pyrrole (Aldrich, $98 \%$ ) was electrochemically polymerised on to FTO (fluorine doped tin oxide) conducting glass plates in the presence of sodiumdodecylbenzenesulfonate (SDBS) (Aldrich, $99 \%$ ). $\mathrm{Ag} / \mathrm{AgCl}$ and $\mathrm{Pt}$ were used as reference electrodes and counter electrodes, respectively. The pyrrole concentration was $0.1 \mathrm{M}$ and the SDBS concentration was $0.05 \mathrm{M}$. The polypyrrole (PPy) film thickness was controlled to be $1 \mu \mathrm{m}$ using a current density of $1 \mathrm{~mA}$ during a period of $240 \mathrm{~s}$ (Vidanapathirana \& Careem, 2006). Single electrode mass was about $0.5 \mathrm{mg}$. The redox capacitors were fabricated by sandwiching a GPE film between two PPy / DBS electrodes.

\section{Characterisation of redox capacitors}

\section{Cyclic voltammetry technique}

Cycling was done by varying the cycling scan rate using a computer controlled potentiostat (Metrohm Autolab M101). The specific capacitance $\left(C_{\mathrm{s}}\right)$ values from this technique were evaluated using the expression,

$C_{\mathrm{s}}=\left(2 \int I \mathrm{~d} v\right) / m \Delta V S$

where, $\int I \mathrm{~d} v$ is the integrated area of the cyclic voltammograms, $m$ is the mass of a single electrode, $\Delta V$ is the potential window and $s$ is the scan rate (Wang et al., 2013). 


\section{Electrochemical impedance spectroscopy}

The impedance data were gathered by using Metrohm autolab impedance analyser in the frequency range of $0.01 \mathrm{~Hz}-0.4 \mathrm{MHz}$. The specific capacitance value was determined using bode plot of real part of the complex capacitance $C^{\prime}(\omega)$ vs frequency. The real capacitance was calculated using the equation,

$C^{\prime}(\omega)=-Z Z^{\prime \prime}(\omega) /\left[\omega|Z(\omega)|^{2}\right]$

where $Z$ " is the imaginary part of the complex impedance, $\omega$ is the angular frequency and $Z$ is the complex impedance (Tey et al., 2016). The maximum value of $C^{\prime}$ $(\omega)$ was used to find the specific capacitance.

\section{Galvanostatic charge discharge test}

The charge-discharge test of the redox capacitor was carried out galvanostatically at $85 \mu \mathrm{A}$ and within the potential window $+0.1 \mathrm{~V}$ and $+0.4 \mathrm{~V}$ for 725 cycles.

Specific energy density $\left(E_{\mathrm{s}}\right)$ and specific power density $\left(P_{\mathrm{s}}\right)$ were calculated using the following equations,

$E_{\mathrm{s}}=C_{s} V^{2} / 2$

and

$P_{\mathrm{s}}=E_{s} / t$

respectively. Here $C_{s}$ is the discharge capacitance, $V$ is the charging potential and $t$ is the discharging time.

\section{RESULTS AND DISCUSSION}

\section{AC conductivity measurements of GPE}

The composition of GPE was initially optimised in order to get a highly conducting, mechanically stable and flexible film for employing as an electrolyte in redox capacitors. Figure 1 shows the variation of conductivity of GPE with NaI concentration at seven different temperatures. It is observed that the conductivity increases with increasing $\mathrm{NaI}$ concentration and the maximum conductivity was obtained at a salt concentration of $0.4 \mathrm{mg}$. The conductivity of a material is governed by several factors, mainly by the concentration as well as the mobility of charge carriers. The initial increase of conductivity with salt concentration is noticeable due to the increase of charge carrier concentration. The subsequent decrease of conductivity after reaching the maximum value is very likely due to the formation of ion pairs in the GPE, which do not facilitate conductivity (Bandaranayake et al., 2016). Also upon increasing the salt concentration, viscosity of the medium may increase. This reduces the mobility greatly. In order to optimise the composition of GPE further, the PVdF concentration was also varied.

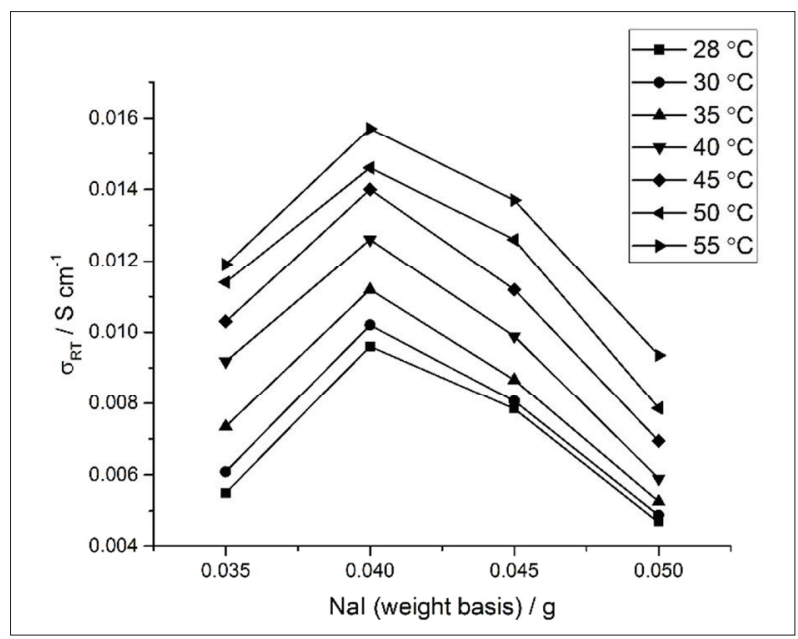

Figure 1: Isothermal graphs for the conductivities of GPEs of four different $\mathrm{NaI}$ concentrations at different temperatures

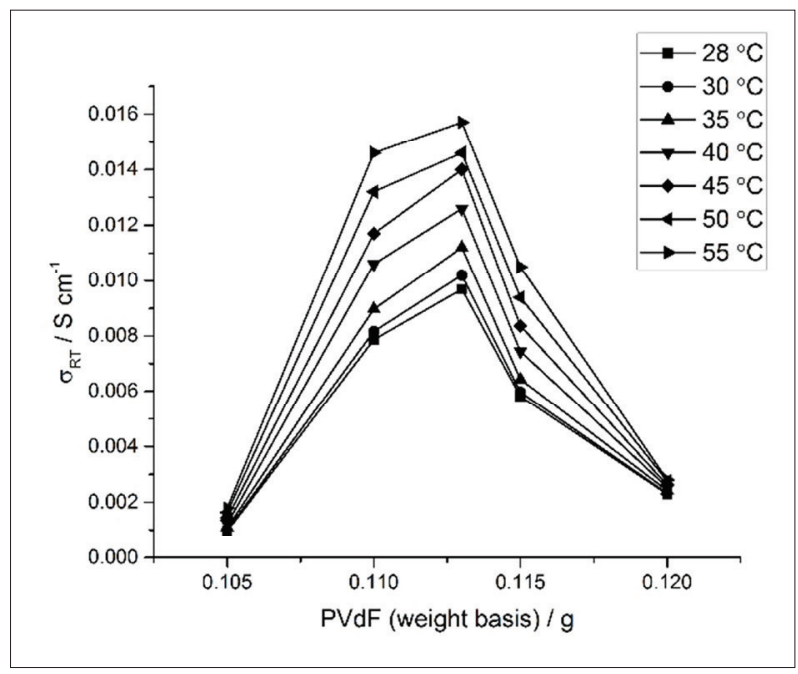

Figure 2: Isothermal graphs for the conductivities of GPEs of five different $\mathrm{PVdF}$ concentrations at different temperatures at the optimum $\mathrm{NaI}$ concentration

The conductivity variation of GPE with the PVdF concentration is shown in Figure 2. When increasing the polymer concentration, assistance for ion motion from the polymer network may increase. This might be 
the reason for initial conductivity enhancement. As the PVdF concentration increases further, viscosity of the system also increases so that there will be a resistance for ion mobility. Due to this, the conductivity may be reduced. The maximum room temperature conductivity is found to be $9.69 \times 10^{-3} \mathrm{Scm}^{-1}$. The composition of GPE that results this conductivity is $1.13 \mathrm{PVdF}: 2.5 \mathrm{EC}$ : $2.5 \mathrm{PC}: 0.4 \mathrm{NaI}$ (by weight). The room temperature conductivity value is very suitable for applications. In addition, this composition results a very thin film.

The plot of $\ln \sigma$ vs 1000/T for optimised GPE is shown in Figure 3. It is observed that the plot is linear

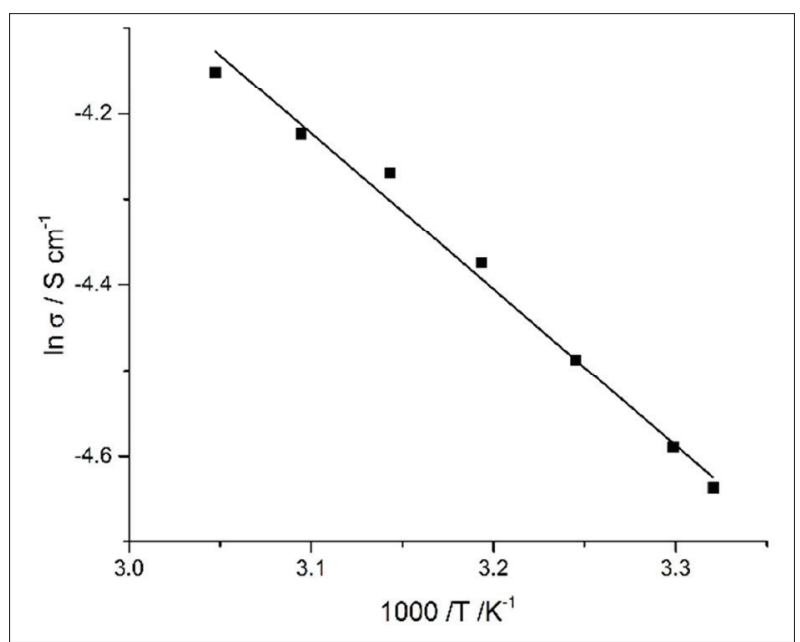

Figure 3: Temperature dependence of the conductivity of the GPE $1.13 \mathrm{PVdF}: 2.5 \mathrm{EC}: 2.5 \mathrm{PC}: 0.4 \mathrm{NaI}$ (weight basis)

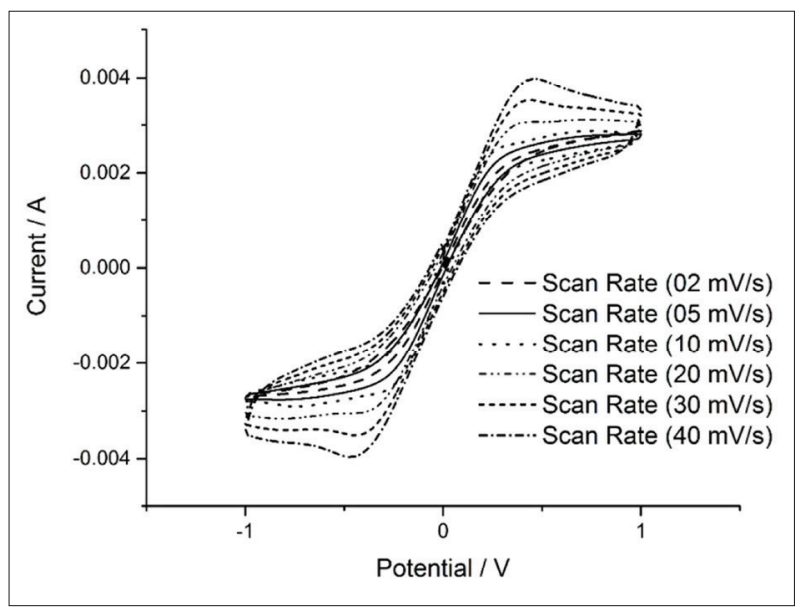

Figure 5: Cyclic voltammograms obtained by varying the scan rates of cycling of the redox capacitor (potential window $+1.0 \mathrm{~V}$ to $-1.0 \mathrm{~V}$ ) thus suggesting that $\sigma$ variation with $\mathrm{T}$ of this GPE follows Arrhenius equation. The Arrhenius behaviour can be explained by the equation,

$\sigma=\sigma_{0} \exp \left(-E_{\mathrm{a}} / k_{\mathrm{B}} T\right)$

where, $\sigma_{0}$ is the pre-exponential factor, $E_{\mathrm{a}}$ is the activation energy for conduction, $K_{\mathrm{B}}$ is the Boltzman constant and $T$ is the absolute temperature. The Arrhenius equation describes ion motion via a hopping mechanism where ions jump into the neighbouring vacant sites and that gives rise to the conductivity.

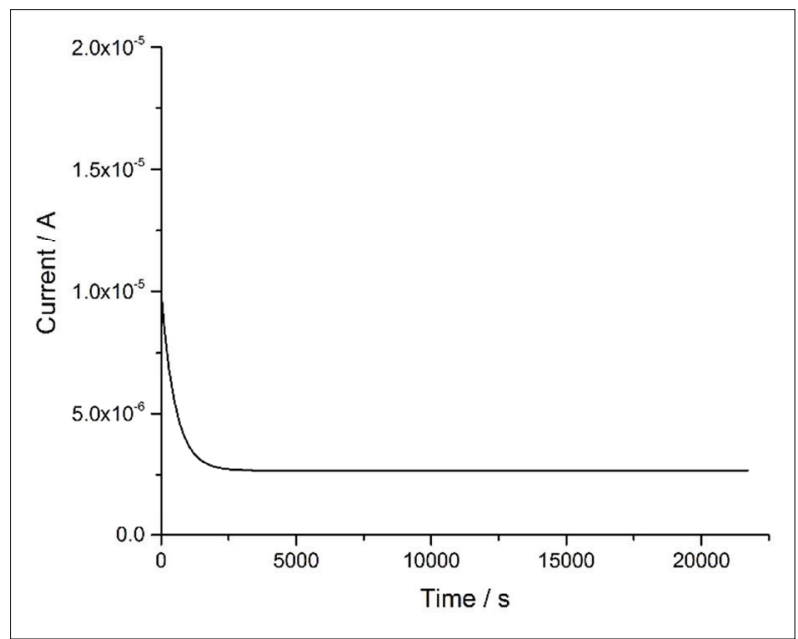

Figure 4: Variation of current through the symmetric cell with two SS electrodes under 1.0 V DC bias potential

\section{Transference number measurements}

Figure 4 shows the graph of current variation through the symmetric cell with time obtained from the polarisation test with SS electrodes. The calculated $t_{i}$ value is 0.84 and this clearly shows that the system under study is a pure ionic conductor.

This optimised composition was used to fabricate the redox capacitors as it was able to obtain a free standing, mechanically stable film with a good ionic conductivity.

\section{Characterisation of redox capacitors}

Cyclic voltammograms (CVs) of the redox capacitor obtained between $-1.0 \mathrm{~V}$ and $+1.0 \mathrm{~V}$ potential range at the scan rates of $2,5,10,20,30$ and $40 \mathrm{mVs}^{-1}$ are shown in Figure 5. All CV patterns exhibit a nearly mirror image symmetry of the current. The oxidation 
and reduction processes of ions during redox processes are symbolised by the peaks in the CVs. The peaks at $0.5 \mathrm{~V}$ are due to oxidation whereas those at $-0.5 \mathrm{~V}$ are due to reduction. During every scan rate, oxidation and reduction have taken place at the identical potential. This is a good indication for the systematic redox processes in the system. Apart from that, it is an evidence to prove that no irreversible or unwanted reactions are occurring in the system.

The specific capacitance of redox capacitors seem to be depending on the scan rate. Figure 6 shows the specific capacitance variation with the scan rate.

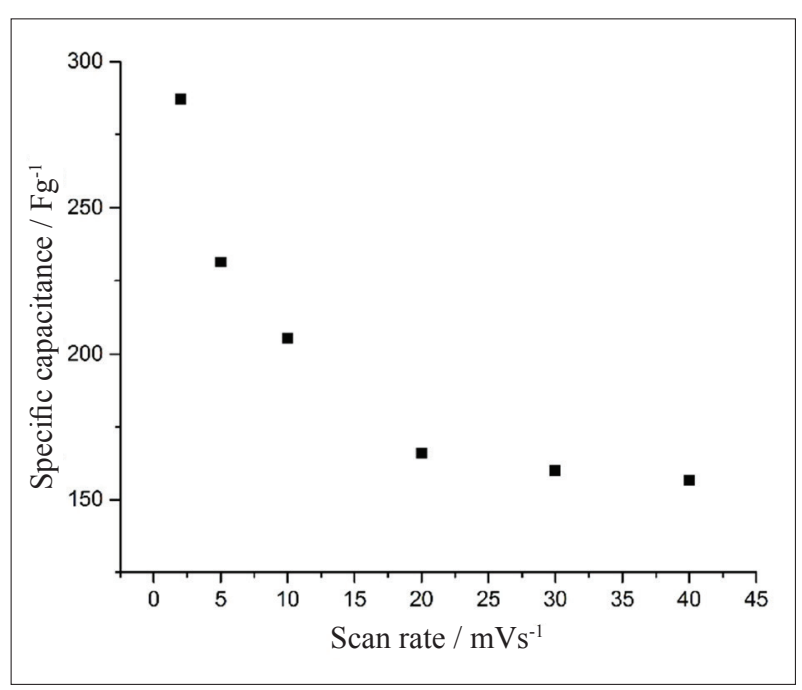

Figure 6: Variation of specific capacitance with the scan rate

When increasing the scan rate, specific capacitance decreased significantly down to $20 \mathrm{mVs}^{-1}$. After that it remained more or less constant. Upon increasing the scan rate, the time needed for the ions to diffuse into the inner surface of the electrode is not sufficient and at higher scan rates, ions may merely penetrate into the inner surface. The reason for the specific capacitance for not pronouncing after $20 \mathrm{mVs}^{-1}$ may be the formation of parallel ionic and electronic conduction paths. Due to this, capacitive property might remain despite the increment of the scan rate.

Figure 7 shows the Bode plots of the real part, $C^{\prime}(\omega)$ of the complex capacitance of the redox capacitor as a function of frequency. The maximum point of the curve associates with the specific capacitance and it is about $3.19 \mathrm{Fg}^{-1}$. This is quite smaller than the value range obtained with the $\mathrm{CV}$ technique. A possible reason may be that in the $\mathrm{CV}$ technique, charging and discharging are being done at different scan rates, which directly affect the specific capacitance. If the cycling were done at a faster scan rate, the two specific capacitance values may be comparable. The reduction of the real component of the capacitance with increasing frequency provides evidence for the transition where the redox capacitor changes from capacitive to resistive (Wang et al., 2011). In the low frequency region, a capacitive property of the electrode material is dominant (Tey et al., 2016). When frequency increases, capacitive behaviour vanishes and resistive behaviour becomes dominant.

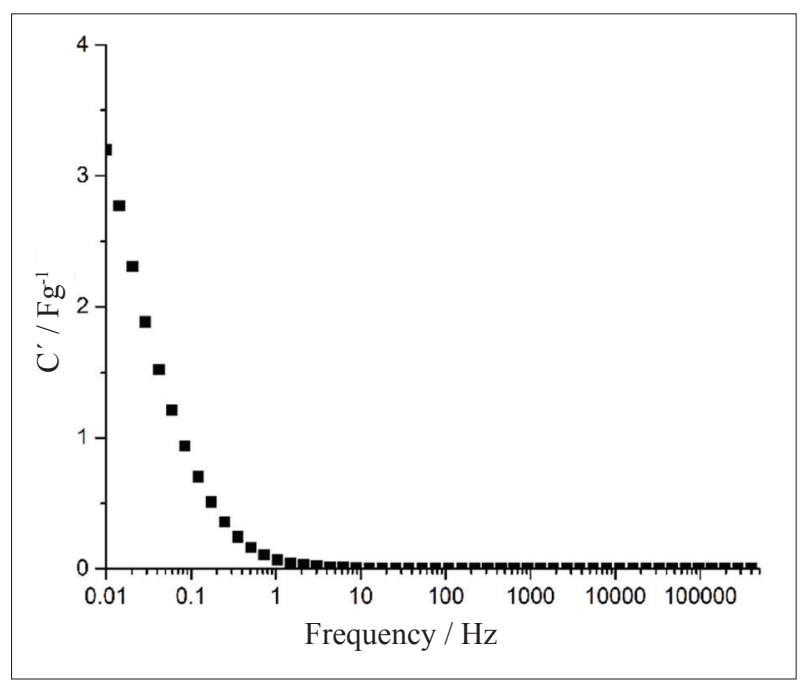

Figure 7: Bode plot for real part of capacitance as a function of frequency in logarithmic scale

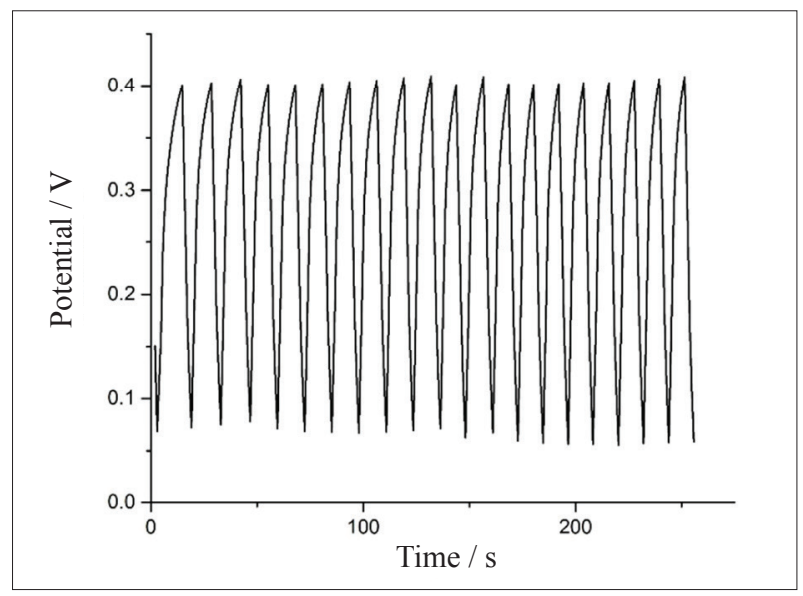

Figure 8: GCD curves obtained for the redox capacitor (charging and discharging current: $85 \mu \mathrm{A}$ ) 
The redox capacitor has also been tested with the galvanostatic charge-discharge method. The typical charge-discharge characteristics of the redox capacitor are shown in Figure 8. The average discharge capacitance was $5.93 \mathrm{Fg}^{-1}$, and specific energy density and specific power density were $0.03 \mathrm{Whkg}^{-1}$ and $0.42 \mathrm{Wkg}^{-1}$, respectively. Somewhat lower energy and power density values may be due to the IR drop of interfacial contact between the electrode and electrolyte.

\section{CONCLUSION}

GPE having the composition $1.13 \mathrm{PVdF}: 2.5 \mathrm{EC}: 2.5$ PC : $0.4 \mathrm{NaI}$ (weight basis) shows the highest room temperature conductivity of $9.69 \times 10^{-3} \mathrm{Scm}^{-1}$ and it was possible to obtain a thin, stable film with the composition. The conduction mechanism is associated with the hopping mechanism. The sample is purely an ionic conductor as evidenced by transference number measurements with SS electrodes. The specific capacitance values obtained from $\mathrm{CV}$ are directly related to the cycling rate. Higher capacitance could be seen at smaller scan rates and when the scan rate increases, specific capacitance reduces down to a certain level and then remains somewhat constant. From EIS technique, the resultant specific capacitance was $3.19 \mathrm{Fg}^{-1}$. The average discharge capacitance of the redox capacitor fabricated from two PPy/DBS electrodes is $5.93 \mathrm{Fg}^{-1}$ from the GCD test, and the corresponding specific energy density is $0.03 \mathrm{Whkg}^{-1}$ and the specific power density is $0.42 \mathrm{Wkg}^{-1}$.

\section{Acknowledgement}

The authors wish to acknowledge the financial assistance provided by the University Grants Commission of Sri Lanka under the innovative research grant scheme 2014 (UGC/VC/DRIC/IRG-2014/WUSL) and the National Science Foundation of Sri Lanka (RG/2014/BS/01 and RG/2015/EQ/07).

\section{REFERENCES}

1. Bandaranayake C.M., Jayathilake Y.M.C.D., Perera K.S., Vidanapathirana K.P. \& Bandara L.R.A.K. (2016). Investigation of a gel polymer electrolyte based on polyacrylonitrile and magnesium chloride for a redox capacitor. Ceylon Journal of Science 45(1): 75 - 82. DOI: https://doi.org/10.4038/cjs.v45i1.7366

2. Gupta A. \& Tripathi S.K. (2013). Effect of anionic size of PMMA based polymer gel electrolytes for redox capacitor. International Journal of Engineering Research and Applications 3(1): $1908-1911$.
3. Jayathilake Y.M.C.D., Perera K.S., Vidanapathirana K.P. \& Bandara L.R.A.K. (2014a). A novel gel polymer electrolyte based on polymethylmethacrylate and copper trifluoromethanesulfonate. Journal of Electroanalytical Chemistry 724: 125 - 129.

DOI: https://doi.org/10.1016/j.jelechem.2014.04.018

4. Jayathilake Y.M.C.D., Perera K.S., Vidanapathirana K.P. \& Bandara L.R.A.K. (2014b). Evaluation of a copper based gel polymer electrolyte and its performance in a primary cell. Sri Lanka Journal of Physics 15: 45 - 51.

5. Jayathilake Y.M.C.D., Perera K.S. \& Vidanapathirana K.P. (2015). Preparation and characterization of a polyacrylonitrile-based gel polymer electrolyte complex with 1methyl-3 propyl imidazolium iodide. Journal of Solid State Electrochemistry 19(8): 2199 - 2203. DOI: https://doi.org/10.1007/s10008-015-2834-7

6. Khanmirzaei M.H., Ramesh S. \& Ramesh K. (2015). Polymer electrolyte based dye-sensitized solar cell with rice starch and 1-ethyl-3-propylimidazolium iodide ionic liquid. Material and Design 85: 833 - 837.

DOI: https://doi.org/10.1016/j.matdes.2015.06.113

7. Pandey G.P., Kumar Y. \& Hashmi S.A. (2010). Ionic liquid incorporated polymer electrolytes for supercapacitor applications. Indian Journal of Chemistry 49: 743 - 751.

8. Singu B.S., Male U., Srinivasan P. \& Pabba S. (2014). Use of surfactant in aniline polymerization with $\mathrm{TiO}_{2}$ to PANI$\mathrm{TiO}_{2}$ for supercapacitor performance. Journal of Solid State Electrochemistry 18: 1995 - 2003.

DOI: https://doi.org/10.1007/s10008-014-2444-9

9. Tey J.P., Careem M.A., Yarmo M.A. \& Arof A.K. (2016). Durian shell-based activated carbon electrode for EDLCs. Ionics 22: 1209 - 1216. DOI: https://doi.org/10.1007/s11581-016-1640-2

10. Tripathi S.K., Jain J., Gupta A. \& Kumari M. (2013). Studies on redox supercapacitor using electrochemically synthesized polypyrrole as electrode material using blend polymer gel electrolyte. Indian Journal of Pure and Applied Physics 51: 315 - 319.

11. Vidanapathirana K.P. \& Careem M.A. (2006). Effect of cathode thickness on the performance of the cell Li/ PAN:EC:PC:LiCF $\mathrm{SO}_{3} / \mathrm{PPy}: \mathrm{DBS}$. Sri Lanka Journal of Physics 7: $29-34$.

12. Wang J., Chen M., Wang C., Wang J. \& Zheng J. (2011). Preparation of mesoporous carbons from amphiphilic carbonaceous material for high-performance electric double layer capacitors. Journal of Power Sources 196: $550-558$.

DOI: https://doi.org/10.1016/j.jpowsour.2010.07.030

13. Wang W., Guo S., Penchev M., Ruiz I., Bozhilov K.N., Yan D., Ozkan M. \& Ozkan C.S. (2013). Three dimensional few layer graphene and carbon nanotube foam architectures for high fidelity supercapacitors. Nano Energy 2: 294 - 303. DOI: https://doi.org/10.1016/j.nanoen.2012.10.001

14. Yu H. et al. (11 authors) (2012). A novel redox mediated gel polymer electrolyte for high-performance supercapacitor. Journal of Power Sources 198: 402 - 407. DOI: https://doi.org/10.1016/j.jpowsour.2011.09.110 